\title{
La generatividad en la vejez y su relación con el bienestar: ¿Quién más contribuye es quien más se beneficia?
}

\author{
Feliciano Villar ${ }^{1 *}$, Olatz López y Montserrat Celdrán ${ }^{2}$ \\ 1 Departamento de Psicología Evolutiva y de la Educación, Universidad de Barcelona \\ 2 Departamento de Metodología de las Ciencias del Comportamiento, Universidad de Barcelona
}

\begin{abstract}
Resumen: El objetivo de la investigación fue la adaptación al español de las escalas de interés y comportamientos generativos de McAdams, así como el estudio de la generatividad en la vejez y su relación con el bienestar. La muestra estuvo compuesta por 165 voluntarios mayores de 65 años que, además de las versiones españolas de las escalas de generatividad, completaron instrumentos para evaluar satisfacción con la vida y orientación al futuro. Los resultados indicaron que la fiabilidad de las versiones españolas de las escalas de generatividad fue aceptable y similar a las originales en inglés. Mientras el interés generativo se relacionó con la satisfacción con la vida, los comportamientos generativos no lo hicieron. Sin embargo, ambas escalas sí se relacionaron con la orientación al futuro, aunque esta relación no se confirmó en un análisis multivariable. Los resultados resaltan la importancia de la generatividad en la vejez y de distinguir entre interés y acción generativa, ya que sus beneficios podrían ser muy diferentes.

Palabras clave: Generatividad en la vejez; bienestar hedónico; bienestar eudaimónico; envejecimiento con éxito.
\end{abstract}

Title: Generativity in older age and its relationship with well-being: Who contributes most is who benefits most?

Abstract: The study was aimed both at adapting the McAdams's generative concern and generative behavior scales into Spanish and at studying generativity in older age and its relationship with well-being. The sample was made up of 165 volunteers aged 65 and over. As well as the Spanish version of the generativity scales, they completed a satisfaction with life scale and an orientation to future scales. Results showed that the Spanish generativity scales had an acceptable level of reliability, similar to the original English versions. Whereas generate concern was related to satisfaction with life, generative behaviors did not. However, both scales were related to orientation to future, although such relationships did not appear in a multivariate analysis. Results emphasize the importance both of generativity in older age and of differentiating generative concern and behaviors, since their benefits could be different.

Key words: Generativity in later life; hedonic well-being; eudaimonic wellbeing; successful aging.

ticipación ha impregnado también la acción política centrada en los mayores (Naciones Unidas, 2002).

En este contexto, nos podemos preguntar hasta qué punto en la vejez se mantienen actividades y motivaciones tradicionalmente asociadas a momentos anteriores de la vida, definiendo nuevos roles para los mayores. El estudio de la generatividad en esta etapa de la vida pretende precisamente dar respuesta a estos interrogantes.

\section{La generatividad en la vejez}

De acuerdo con la teoría del desarrollo de Erik Erikson (1950), la generatividad es el reto al que la persona se enfrenta en la mediana edad. Se define como el interés por guiar y asegurar el bienestar de las siguientes generaciones y, en último término, por dejar un legado que nos sobreviva. La generatividad se puede expresar a partir de actividades como la crianza de los hijos, el cuidado a personas dependientes, la formación de los jóvenes, la producción de bienes y servicios o el compromiso social y la participación cívica y política. En cualquier caso, implica contribuir al bien común de los entornos en los que las personas participan (la familia, la empresa, la comunidad, etc.), para reforzar y enriquecer las instituciones sociales, asegurar la continuidad entre generaciones o plantear mejoras sociales.

Tras unas décadas de relativo olvido, la generatividad es reintroducida en los estudios sobre el desarrollo en la adultez por Dan McAdams, quien diferencia entre el interés generativo, definido como una actitud favorable hacia cuestiones generativas, y la acción generativa, o puesta en marcha y concreción en comportamientos de esa actitud favorable (McAdams y Logan, 2004; McAdams, Hart, y Maruna, 1998). Este mismo autor desarrolló instrumentos para eva-
Feliciano Villar, Departamento de Psicología Evolutiva y de la Educación, Universidad de Barcelona, Passeig de la Vall d'Hebron 171, Barcelona 08035 (España). E-mail: fvillar@ub.edu 
luar esas dos dimensiones de la generatividad que han sido utilizadas en numerosas investigaciones posteriores, aunque desgraciadamente, no han sido validadas todavía en nuestro entorno. Como veremos, este es uno de los objetivos de nuestro estudio.

A pesar de que los trabajos de McAdams seguían restringiendo la generatividad a la mediana edad, en las últimas décadas ha aparecido un interés por comprobar si los intereses y acciones generativas se extienden también a la vejez, y qué papel desempeñan en las personas mayores. El propio Erikson en sus últimos escritos (Erikson, Erikson y Kivnick, 1986) planteó el concepto de grand generativity, que trata de reconocer las diversas formas en las que los mayores ayudan a otros (como padres, como abuelos, como amigos, como mentores, etc.) a la vez que aceptan ayuda y expresan su interés por perpetuar conocimientos y valores en las futuras generaciones.

Desde un punto de vista empírico, la asociación estricta entre generatividad y mediana edad también es dudosa. Por ejemplo, tras incrementarse en la transición de la juventud a la mediana edad, el interés y las actividades generativas en general se mantienen en la vejez, tanto cuando se comparan trasversalmente diversos grupos de edad (Sheldon y Kasser, 2001; Zucker, Ostrove, y Stewart, 2002) como cuando las personas evalúan, retrospectivamente, la generatividad en diferentes momentos de su vida (Miner-Rubino, Winter, y Stewart, 2004). Así, la generatividad parece estar detrás de las contribuciones de los mayores tanto en ámbitos familiares como comunitarios (Kleiber y Nimrod, 2008; Thiele y Welan, 2008; Warburton y Gooch, 2007).

\section{Generatividad y bienestar en la vejez}

El concepto de generatividad en la vejez no es el único que enfatiza la participación y contribuciones de los mayores en la sociedad. Otros, como el de envejecimiento activo (Fernández-Ballesteros, 2009; Organización Mundial de la Salud, 2002) o envejecimiento productivo (Caro y Sánchez, 2005; Ranzijn, 2002) son similares en este sentido. Sin embargo, a diferencia de ellos, la generatividad también implica un componente de desarrollo personal, de crecimiento y de madurez. Así, la actividad generativa en la vejez no implica sólo contribuir a la mejora y sostenimiento de los contextos en los que participan, sino también una actividad que da sentido y propósito a la vida de los mayores. Contribuir aporta no sólo beneficios para los demás, sino también recompensas personales.

Por ello la generatividad en la vejez podría ser una importante fuente de bienestar en las últimas décadas de la vida y ser un importante ingrediente del envejecimiento satisfactorio (Fisher, 1995; Villar, en prensa). Diversos estudios han tratado de indagar en la relación entre bienestar y generatividad, generalmente con resultados contradictorios. Así, McAdams, de St. Aubin y Logan (1993) o Grossbaum y Bates (2002), utilizando muestras de mediana edad, encontraron que mientras el interés generativo sí mantenía una rela- ción positiva con la satisfacción con la vida, las acciones generativas no. Con una muestra de personas mayores, Cheng (2009) obtuvo que las diferencias entre interés y acción generativa se mantenían, aunque en este caso las acciones generativas sí se relacionaban, aunque moderadamente, con el bienestar. En el estudio de Cheng, el impacto de la generatividad sobre el bienestar dependía de hasta qué punto las contribuciones de los mayores eran valoradas por los demás.

Los autores atribuyen estas diferencias entre interés y actividad generativa a las diferentes oportunidades que las personas pueden tener para llevar a la práctica su interés generativo, en función de diversas circunstancias vitales o variables como el género, el nivel educativo o el estado civil (McAdams et al., 1998). Además, quizá estar implicado en acciones generativas implica mayores dificultades y sinsabores que simplemente expresar interés y preocupación por los demás (Warburton, McLaughin, y Pinsker, 2006).

Sin embargo, el impacto de la generatividad en el bienestar también podría variar en función del modo en el que se entienda el bienestar. En este sentido, algunos autores diferencia entre bienestar 'hedónico', referido a sentimientos presentes de placer o displacer (que se concretan en medidas de felicidad o de satisfacción con la vida) y bienestar 'eudaimónico', centrado en el esfuerzo por conseguir objetivos a largo plazo, la realización del propio potencial y sentirse a uno mismo en desarrollo (Ryan y Deci, 2001; Waterman, 1993). En este sentido, el bienestar eudaimónico en ocasiones implica esfuerzos y sacrificios que pueden dañar los sentimientos presentes de placer (es decir, el bienestar hedóni$\mathrm{co})$.

Diversas investigaciones han mostrado como el bienestar de tipo eudaimónico (al contrario que el hedónico) tiende a disminuir con la edad (p.e., Ryff, 1995). Sin embargo, la generatividad puede aportar un conjunto de objetivos con sentido a los mayores, orientados a contribuir al bienestar de los demás y a dejar un legado que vaya más allá de uno mismo y de las recompensas inmediatas. Si esto es así, deberíamos encontrar una relación positiva entre generatividad y bienestar eudaimónico. Esta relación sería particularmente intensa si el interés generativo es capaz de transformarse en acciones generativas.

\section{Objetivos}

El estudio que se plantea presenta tres objetivos principales:

1. Desarrollar una versión española de las escalas de generatividad de McAdams (interés generativo y acciones generativas) y aportar datos que contribuyan a su validación con muestras de personas mayores.

2. Determinar el grado de interés generativo y los comportamientos generativos más frecuentes entre las personas mayores, así como la relación del interés y comportamientos generativos con el género, la edad, el nivel de estudios, el estatus marital, la salud y con la participación en asociaciones y cuidado a nietos o personas dependientes. 
3. Determinar la relación entre interés y comportamientos generativos con las dimensiones hedónicas y eudaimónicas del bienestar. En este sentido, se espera que el interés generativo tenga una relación particularmente intensa con el bienestar hedónico, mientras que el bienestar eudaimonico se relacione por igual con ambos aspectos generativos, o incluso en mayor medida con los comportamientos generativos que con el interés generativo. Adicionalmente, se pretende estudiar el peso predictivo en esas dos dimensiones de bienestar (hedónico y eudaimónico) del interés y comportamientos generativos, así como del género, la edad, el nivel de estudios, el estatus marital, la salud, la participación en asociaciones y el cuidado de nietos o personas dependientes.

\section{Método}

\section{Participantes}

La muestra estaba compuesta de 165 personas de entre 65 y 100 años $(M=73.55 ; D T=7.07)$. El $60 \%$ de ellas $(99$ participantes) fueron mujeres y el $40 \%$ hombres.

En cuanto al estado civil, había un predominio de personas casadas $(56.4 \%)$, viudas $(37 \%)$ y una minoría de solteras, separadas o divorciadas $(6.7 \%)$. Sólo cuatro de los participantes $(2.4 \%)$ manifestaron no tener hijos, mientras que nueve $(5.5 \%)$ no tenían nietos. Respecto al nivel educativo, casi la mitad tenía estudios primarios (46.1\%), una quinta parte educación secundaria $(21.8 \%)$, un porcentaje similar estudios superiores $(21.2 \%)$ y sólo una minoría no tenía estudios $(10.9 \%)$. En cuanto a la convivencia, la mayoría no vivía solo $(67.9 \%)$, de hecho aproximadamente la mitad de la muestra (56.4\%) convivía en pareja, casi una cuarta parte con algún hijo $(22.4 \%)$ y unos pocos con otros familiares $(8.5 \%)$, nietos $(4.8 \%)$ u otras personas fuera del ámbito familiar $(1.8 \%)$. Las relaciones más frecuentes, es decir, aquellos con los que se mantenía un contacto diario o semanal, las mantenían con los hijos (82.6\%), los vecinos y amigos $(74.1 \%)$ y los nietos $(60.9 \%)$.

Respecto a la participación en asociaciones, un 24.9\% participaba con alguna o mucha frecuencia en asociaciones religiosas, un $17.6 \%$ en centros de mayores, un $13.5 \%$ en asociaciones de vecinos, un $6.6 \%$ en organizaciones de voluntariado y sólo un $3 \%$ en asociaciones políticas o sindicales. En cuanto cuidado, un $31.3 \%$ cuidaba con frecuencia (semanalmente o más) a nietos y un $23.4 \%$ a familiares adultos dependientes.

\section{Instrumentos}

Los participantes completaron un cuestionario en el que se les instaba a proporcionar diversos datos de tipo sociodemográfico, tales como el género, la edad, el estado civil (con cuatro opciones, casado, soltero, viudo y separado/divorciado) y nivel de estudios (con cuatro niveles: sin estudios, solo estudios primarios, estudios secundarios y es- tudios universitarios). Se incluyeron cinco ítems para valorar la participación social en diversos tipos de instituciones y asociaciones (asociaciones de vecinos, religiosas, centros de mayores, instituciones de voluntariado y asociaciones políticas o sindicales). Para valorar la frecuencia también se utilizó un formato de respuesta de cuatro puntos, que iba de 'nunca' (0) a 'muy a menudo' (3). También se preguntó si tenían como responsabilidad el cuidado de nietos o familiares dependientes. En los dos casos se utilizó una escala de cuatro puntos que iba de 'nunca' a 'diariamente'. Finalmente, se incluyeron dos preguntas relativas a la salud: en una se pedía que valorase su estado de salud en una escala de cuatro puntos, de 'excelente' (3) a 'mala' (0), en la otra que indicase cuántos medicamentos diferentes tomaba a diario.

Tras completar el cuestionario, los participantes completaron las siguientes escalas:

Escala de Generatividad Loyola (EGL) - La escala LSG (Loyola Generativity Scale), desarrollada por McAdams y de St. Aubin (1992) y destinada a evaluar el interés generativo en población adulta, se tradujo al español. Esa primera versión en español fue, a su vez, traducida al inglés por una persona bilingüe. Se examinaron las discrepancias entre versiones para llegar a una versión definitiva, que contaba de 20 ítems con un formato de respuesta tipo Likert de cuatro puntos $(0$ "nunca", 1 "ocasionalmente", 2 "muy a menudo", 3 "casi siempre”). Al igual que la versión en inglés, seis ítems (los mismos) se formularon de manera invertida. La puntuación total podía tener un rango de 0 a 60 puntos, indicando un mayor interés generativo a mayor puntuación total en dicha escala. De acuerdo con McAdams y de St. Aubin (1992), la escala LGS demostró una alta consistencia interna (alfa de Cronbach de .83) y una fiabilidad tes-retest adecuada (.73 en un intervalo de tres semanas), así como una validez de constructo derivada de la correlación positiva con otras medidas de generatividad (McAdams et al., 1993). En el anexo 1 se incluye la versión final de la EGL utilizada en el estudio.

Escala de Comportamiento Generativo (ECG) - Siguiendo el mismo procedimiento que la escala anterior, se tradujo al español la Generative Behavioral Checklist (GBC), destinada a evaluar la frecuencia de conductas generativas en población adulta. De la escala original de 65 ítems (49 actos generativos y 16 neutros), y con el fin de obtener un instrumento más corto y adaptado a las personas mayores, se seleccionaron los 29 que más relevancia podrían tener en la vejez, eliminando además los ítems neutros. La escala resultante se antecedió de la instrucción 'Indique cuántas veces en los últimos dos meses ha realizado cada una de las siguientes actividades', equivalente a la de la escala en inglés. Al igual que la versión original, los ítems se contestaban utilizando un formato de respuesta de 3 puntos (0 "nunca", 1 "una vez", 2 "dos o más veces”). La puntuación total podía tener un rango de 0 a 58 puntos, en que a mayor puntuación indicaba mayor comportamiento generativo del mayor. La GBC original en inglés demostró validez de constructo al correlacionar positivamente con la LGS $(r=.53)$ y con otras medidas de generatividad (McAdams y de St. Aubin, 1992; McA- 
dams et al., 1993). En el anexo 2 se incluye la versión final de la ECG utilizada en el estudio.

Escala Satisfacción Vital (ESV) - Se aplicó la Satisfaction with Life Scale de Diener, Emmons, Larsen y Griffin (1985), en concreto la adaptación española realizada por Pons, Atienza, Balaguer y García-Merita (2000) con personas mayores. La escala consta de cinco ítems orientados a obtener una evaluación general que la persona realiza sobre su propia vida, con lo que puede considerarse una medida de bienestar hedónico (Deci y Ryan, 2008). Los ítems se contestaron en un formato de respuesta tipo Likert de 4 puntos (0 "muy en desacuerdo", 1 "más bien en desacuerdo", 2 "más bien de acuerdo"; 3 "muy de acuerdo"). La puntuación total tenía un rango de 0 a 15 puntos, en el que a mayor puntuación indicaba una mayor satisfacción con la vida. El instrumento poseía unas buenas propiedades psicométricas, con una alfa de Cronbach de .87 en el caso de la escala original en inglés (Diener et al., 1985) y de .82 en la versión española.

Escala Ryff Abreviada (ERA) - Se aplicó la versión breve de las Ryff's Well-being Scales (Ryff, 1989), traducida y validada en español con muestras de personas mayores por Villar, Triadó, Celdrán y Solé (2010). En concreto, se aplicó la subescala de siete ítems 'Orientación al futuro'. Esta subescala recoge ítems de las dimensiones Propósito en la Vida y Crecimiento Personal de la escala original de Ryff, que se consideran buenos indicadores del bienestar eudaimónico (Ryff, 1995). La subescala aplicada constaba de siete ítems con un formato de respuesta tipo Likert de cuatro puntos $(0$ "muy en desacuerdo", 1 "más bien en desacuerdo", 2 "más bien de acuerdo", 3 "muy de acuerdo"). La puntuación total podía tener un rango de 0 a 21 puntos, en el que a mayor puntuación mostraría un mayor bienestar eudaimónico. La subescala, con población mayor, ha demostrado tener una aceptable consistencia interna, con una alfa de Cronbach de .73 .

\section{Procedimiento}

Los datos se recogieron mediante la auto-aplicación individual de los cuestionarios a personas mayores de 65 años de las ciudades Barcelona y Tarragona, y sus áreas metropolitanas.

La obtención de los participantes se hizo a partir de estudiantes de Psicología y Educador Social de la Universidad de Barcelona y la Universidad Rovira i Virgili. A cada estudiante se le dieron dos sobres cerrados, cada uno de ellos con los instrumentos que las personas mayores debían rellenar. La tarea del estudiante consistía en ofrecer, y recoger al cabo de una semana, los sobres con los instrumentos dos personas mayores de 65 años de su entorno. Los estudiantes realizaron esta tarea voluntariamente y completarla no implicaba ningún beneficio para ellos.

El sobre incluía, junto a los instrumentos, una carta explicando el propósito del estudio y su carácter anónimo y confidencial. En esa carta se incluía una aceptación a partici- par voluntariamente en el estudio que tenía que ser firmada por el participante.

De los 560 sobres entregados, se recogieron 322. Una vez descartados aquellos cuestionarios incompletos o que no tenían firmado el consentimiento informado, se obtuvieron un total de 265 cuestionarios, la mayoría de ellos eran mujeres. Para conseguir cierto equilibro entre hombres y mujeres, o al menos una distribución similar a que presentaban otros estudios sobre generatividad en la vejez (McAdams y de St. Aubin, 1992; McAdams et al., 1993) se realizó una selección aleatoria de las mujeres de la muestra para obtener cierto equilibro (60\% mujeres y $40 \%$ hombres) en el género, similar a estudios realizados con la EGL y la ECG en muestras de personas mayores. Fruto de esta selección, la muestra definitiva estuvo compuesta por 165 personas.

Para asegurarse de que los participantes procedentes de Barcelona (133) y de Tarragona (32) eran equivalentes en las variables principales del estudio, se realizaron ANOVAs, obteniéndose valores $F$ en un rango desde 0.13 a 2.21 , y que no fueron estadísticamente significativas en ningún caso.

\section{Resultados}

\section{Escala de Generatividad Loyola}

Para validar la adaptación de la EGL en personas mayores se procedió primero a realizar un análisis factorial exploratorio (AFE) mediante la técnica de componentes principales (CP) y rotación varimax. La medida Kaiser-Mayer-Olkin $(K M O=.71)$ verificó la adecuación muestral y la prueba de esfericidad de Barlett $\left(\chi^{2}(190)=593.69 ; p<.001\right)$ indicó que la correlación entre ítems era suficiente para aplicar dicha técnica de análisis. Se obtuvieron dos componentes con auto-valores mayores a 1 (con saturaciones factoriales superiores a .4), cuya combinación explicaba el $29.1 \%$ de la variancia total: el componente de Generatividad Positiva (items $1,4,6,8,10,12,17,18,19,20)$, que explicaba el $18.8 \%$ de la variancia total, junto con el componente de Dudas generativas (items revertidos $2,13,14,15$ ) que explicaba el $10.2 \%$. Se podían haber extraído entre 2 ó 3 componentes según el gráfico de sedimentación, pero se especificó que fueran dos los factores por dos razones: la convergencia de nuestros datos con los resultados de la escala original de McAdams y de St. Aubin (1992), quienes identifican exactamente los mismos dos componentes, y porque el punto de inflexión más claro aparece después del segundo componente (y el tercero apenas explicaba un 1.5\% más de la variancia total). La consistencia interna se analizó mediante el coeficiente alfa de Cronbach, que a nivel general obtuvo un valor de $.89 \mathrm{y}$, en particular obtuvo un .78 para el primer factor y un .62 para el segundo factor. Hay que tener en cuenta, sin embargo, que éste último tenía un número de ítems menor. El nivel de consistencia interna de la escala es similar, o incluso ligeramente superior, al reportado por McAdams y St. Aubin (1992) para la escala original. 
La puntuación media en la EGL fue de 35,6 (DT = 7.79), e iba desde una puntuación mínima de 16 a una máxima de 54. Esta media era ligeramente inferior a la obtenida por McAdams y de St. Aubin (1992) con una muestra de mediana edad, pero superior a la reportada por Cheng (2009) con una muestra de personas mayores. Una vez invertidas las puntuaciones de los ítems negativos, en seis de ellos se obtuvieron puntuaciones medias superiores a dos. Fueron los ítems 15 ('Me siento como si no hubiese hecho nada valioso que pudiera servir a otras personas', $M=2.51 ; D T=0.88), 2$ ('Siento que nadie me necesita', $M=2.43 ; D T=0.89$ ), 14 ('En general, lo que hago no tiene un efecto positivo sobre los demás'; $M=2.29 ; D T=0.95$ ), 4 ('Siento que importo a muchas personas', $M=2.28 ; D T=0.85$ ) y 13 ('Siento que nada de lo que he hecho sobrevivirá después de que muera', $M=2.21 ; D T=0.99)$.

Para estudiar la relación entre el interés generativo (medido a partir de las puntuaciones en la EGL) y la edad se llevó a cabo una correlación de Pearson, que mostró que ambas variables no estaban relacionadas $(r(159)=.07, p=$ .35). La misma prueba fue aplicada para conocer la relación entre interés generativo y estudios, obteniéndose en este caso un valor no significativo estadísticamente $(r(159)=.08, p$ $=.30)$. Para comprobar si las puntuaciones en el EGL eran diferentes entre mujeres y hombres, se realizó una prueba $t$ de Student para grupos independientes, en la que no se encontraron diferencias significativas $(t(157)=0.16, p=.87)$. La misma prueba estadística se utilizó para comparar las medias en las puntuaciones en la EGL entre los casados y los no casados, sin obtenerse tampoco diferencias significativas $(t(163)=0.91 ; p=.39)$.

La relación entre el interés generativo y la participación en asociaciones, indicada por una correlación lineal de Pearson, no fue estadísticamente significativa $(r(156)=.12, p$ $=.13$ ). El interés generativo, utilizando la misma prueba, tampoco estaba relacionado con la frecuencia con la que se ofrecían cuidados a nietos o personas dependientes $(r$ (159) $=.12, p=.13$ ). Utilizando la misma prueba, se comprobó si el interés generativo estaba relacionado con la salud. Ni en el caso de la salud $\operatorname{subjetiva}(r(165)=-.01, p=.92)$ ni en el de el número de medicamentos $(r(165)=-.09, p=.22)$ las relaciones con el interés generativo fueron estadísticamente significativas.

\section{Escala de Comportamiento Generativo}

La validación de la adaptación de la ECG a la población mayor también se realizó mediante un AFE con el análisis de CP y rotación varimax. La medida KMO (.69) y la prueba de Barlett $\left(\chi^{2}(406)=968.84 ; p<.001\right)$ indicaron la adecuación para aplicar esta técnica de análisis. Se obtuvieron cuatro componentes con auto-valores mayores a 1 (con saturaciones factoriales superiores a 0.4 ), cuya combinación explicaba el $36,4 \%$ de la variancia: el componente de Cuidado (ítems 3, 5, 9 y 20) con el 8,58\% de la variancia, el componente de Colaborar (items 13, 14, 15, 24 y 26) referente al
$7.33 \%$ de la variancia explicada, el componente de Donar (ítems 4, 11, 21 y 22) con el 7.31\% de la variancia y el componente Voluntariado (ítems 10 y 28) que explicaba el 7.06\% de la variancia total. Se podían extraer entre 2 ó 4 componentes según el gráfico de sedimentación, pero se especificó que fueran cuatro, dado que el punto de inflexión más claro parece estar después del cuatro componente (el quinto apenas explicaba un $1.8 \%$ de la variancia total) y el significado de los componentes resultados era fácilmente interpretable. De hecho, la estructura de componentes reproduce la obtenida por McAdams y de St. Aubin (1992). La consistencia interna se analizó mediante el alfa de Cronbach, que a nivel general obtuvo un valor de .81 y un .63, .64, .64 y .69 para los componentes respectivos, por lo que la ECG tenía una alta fiabilidad general, aunque sólo moderada en cada componente.

Posteriormente, se analizó la respuesta media de la muestra a la ECG $(M=28.11 ; D T=9.64$; con una puntuación mínima de 1 y máxima de 52). Las conductas generativas más frecuentes entre los participantes, con puntuaciones medias superiores a 1.5 , fueron las siguientes: 'He cuidado del bienestar de mi familia' $(M=1.80, D T=0.54)$, 'He atendido a las necesidades de personas que me rodean' ( $M=$ $1.59, D T=0.74)$, 'He cuidado de plantas o animales domésticos' $(M=1.58, D T=0.76)$, 'He transmitido valores a personas más jóvenes' $(M=1.50, D T=0.77)$ y 'He realizado acciones que ayudan a mejorar el medio ambiente (reciclar, plantar un árbol, recoger basura de la calle, etc.)' $(M=1.50$, $D T=0.81)$

Mediante una prueba $t$ de Student para muestras relacionadas, se obtuvo que la puntuación media en la ECG fue significativamente menor que la obtenida en la EGL ( $t$ (158) $=10.54 ; p<.001)$.

Para conocer la relación entre la edad y el comportamiento generativo, medida a partir de las puntuaciones en la ECG, se llevó a cabo una correlación lineal de Pearson, en la que se obtuvo que las personas más mayores tendían a mostrar menos comportamientos generativos $(r(159)=-.21 ; p<$ .01). También se correlacionó la puntuación en la ECG con los estudios, para comprobar su relación, obteniéndose un valor estadísticamente no significativo $(r(159)=.09 ; p=$ .23). Para determinar la posible influencia del género en los comportamientos generativos, se compararon puntuaciones de hombres y mujeres mediante la prueba $t$ de Student para muestras independientes, cuyo resultado indicó que las diferencias no fueron significativas $(t(157)=-0.37, p=.80)$. Utilizando la misma prueba, tampoco resultaron significativas las comparaciones entre las medias de los casados y no casa$\operatorname{dos}(t(163)=1.38, p=.17)$.

A diferencia de lo que sucedía con las puntuaciones en la EGL, la puntuación en la ECG sí estuvo significativamente relacionada, utilizando el valor de correlación lineal de Pearson, tanto con la participación en asociaciones $(r(162)=.21$; $p<.05)$ como con la frecuencia en la que ofrecían cuidados $(r(160)=.31 ; p<.001)$. Esta misma prueba se aplicó para estudiar la relación de la EGL con la salud subjetiva $(r(160)$ 
$=.03 ; p=.66)$ y con el número de medicamentos $(r(160)=$ $.05 ; p=.50)$, no siendo significativa la relación en ninguno de los dos casos.

Por último, la correlación lineal de Pearson entre la EGL y la ECG también fue estadísticamente muy significativa $(r$ $(159)=.49 ; p<.001)$, situándose en niveles similares a las correlaciones encontradas con las escalas originales en inglés (McAdams y de St. Aubin, 1992).

\section{Generatividad y bienestar}

En la Tabla 1 podemos observar las correlaciones entre el interés generativo y las medidas de bienestar utilizadas en el estudio (satisfacción con la vida y orientación al futuro).

Tabla 1. Correlaciones de Pearson y estadísticos descriptivos. Los valores de consistencia interna alfa de Cronbach figuran en la diagonal principal.

\begin{tabular}{lllll}
\hline & 1 & 2 & 3 & 4 \\
\hline 1. Interés generativo & .89 & & & \\
2. Comportamiento generativo & $.49 * *$ & .81 & & \\
3. Satisfacción con la vida & $.38^{* *}$ & .10 & .78 & \\
4. Orientación al futuro & $.27^{* *}$ & $.32^{* *}$ & .09 & .73 \\
& & & & \\
Rango & $0-60$ & $0-58$ & $0-15$ & $0-21$ \\
$M$ & 35.60 & 28.11 & 11.03 & 9.87 \\
DT & 7.79 & 9.64 & 2.85 & 4.44 \\
\hline
\end{tabular}

Se observa que, mientras la satisfacción con la vida y la orientación al futuro se relacionan ambas de manera significativa con el interés generativo, el comportamiento generativo sólo está relacionado con la orientación al futuro, pero no con la satisfacción con la vida.

Para comprobar el peso predictivo en los distintos tipos de bienestar de diferentes variables, tanto relacionadas con la generatividad como de carácter sociodemográfico, se llevaron a cabo análisis de la regresión múltiple. Para estos análisis se utilizó el método de introducción. En cuanto la satisfacción con la vida, evaluada mediante la ESV, el interés generativo resultó ser un predictor muy significativo, obtenido una correlación parcial (eliminando los posibles efectos del resto de variables) elevada, de .45 . El nivel de estudios también alcanzó la significación estadística como predictor, aunque en niveles inferiores, mientras que el resto de variables, incluida la medida de comportamientos generativos, no eran predictores significativos de la satisfacción con la vida (ver Tabla 2).

Por lo que respecta a la orientación al futuro, en este caso ni el interés ni los comportamientos generativos fueron predictores estadísticamente significativos. La edad, sin embargo, sí lo fue: a mayor edad, se obtenía una menor orientación al futuro. La salud subjetiva, aunque en menor medida, también fue un predictor significativo (ver Tabla 3).
Tabla 2. Predictores de la satisfacción con la vida.

\begin{tabular}{lccrrr}
\hline & $R^{2}$ & $B$ & $C I B$ & \multicolumn{2}{c}{ Beta $\begin{array}{c}\text { Corr. } \\
\text { parcial }\end{array}$} \\
\hline Satisfacción con la vida & .365 & & & & \\
$\quad$ Interés generativo & .18 & {$[.85-2,72] .47 * *$} & .45 \\
Compor. generativo & -.04 & {$[-.11-.02]$} & -.13 & -.14 \\
Género & -.63 & {$[-1.85-.59]$} & -.12 & .13 \\
Edad & -.01 & {$[-.11-.10]$} & -.14 & .11 \\
Estado civil & -.45 & {$[-1.49-.55]$} & -.08 & -.07 \\
Estudios & .62 & {$[2.60-.11]$} & $.29 *$ & .30 \\
Salud subjetiva & -.32 & {$[-1.12-.47]$} & -.09 & -.09 \\
Num. Medicamentos & -.07 & {$[-.28-.12]$} & -.09 & -.09 \\
Participación asociaciones & .15 & {$[-.07-.38]$} & .15 & .16 \\
Cuidados & -.08 & {$[-1.85-.59]$} & -.05 & -.05 \\
\hline Nota: ${ }^{* *} p<.001 ; * p<.01$ & & & & &
\end{tabular}

Tabla 3. Predictores de la orientación al futuro.

\begin{tabular}{|c|c|c|c|c|c|}
\hline & $\mathrm{R}^{2}$ & $B$ & IC B 95\% & Beta & $\begin{array}{l}\text { Corr. } \\
\text { Parcial }\end{array}$ \\
\hline Orientación al futuro & .407 & & & & \\
\hline Interés generativo & & .09 & {$[-.00-.18]$} & .15 & .17 \\
\hline Compor. Generativo & & .02 & {$[-.05-.10]$} & .05 & .05 \\
\hline Género & & 1.93 & {$[.38-3.47]$} & $.21 *$ & .21 \\
\hline Edad & & -.30 & {$[-.41--.20]$} & $-.49 * *$ & - -.46 \\
\hline Estado civil & & -.13 & {$[-1.58-1.32]$} & -.01 & -.02 \\
\hline Estudios & & .06 & {$[-.52-.64]$} & .02 & .02 \\
\hline Salud subjetiva & & 1.22 & {$[-2.14--.32]$} & $-.21 *$ & -.23 \\
\hline Num. Medicamentos & & -.03 & {$[-.25-.18]$} & -.02 & -.03 \\
\hline Participación asociaciones & & .04 & {$[-.17-.25]$} & .03 & .03 \\
\hline Cuidados & & -.02 & {$[-.47-.43]$} & -.01 & -.01 \\
\hline
\end{tabular}

\section{Discusión}

Los resultados obtenidos muestran que las versiones en español de las escalas de interés generativo y de comportamientos generativos presentan unas propiedades aceptables y equivalentes a las versiones originales en inglés en las que se basan. Evalúan aspectos sin duda relacionados, pero no equivalentes, ya que presentan un perfil de correlaciones con otras variables diferentes, lo que indica que tanto sus determinantes como sus consecuencias tienen cierto grado de especificidad. Por ejemplo, mientras el interés generativo se mantiene relativamente estable con la edad, los comportamientos generativos sí parecen ser menos frecuentes en las personas de mayor edad. De manera similar, como veremos, sus relaciones con el bienestar son también diferentes.

En todo caso, estos hallazgos también sugieren que la generatividad es un fenómeno frecuente y con sentido en la vejez, ya sea como actitud o predisposición (interés generativo) o como actividades concretas (comportamiento generativo). De hecho, las puntuaciones en interés generativo son comparables a las obtenidas en otros estudios con muestras de personas de mediana edad (ver, por ejemplo, McAdams et al., 1993) e incluso superiores a los que otros autores han encontrado en muestras de personas mayores (Cheng, 2009). En este último caso, sin embargo, hay que tener en cuenta que Cheng obtuvo sus datos de una muestra procedente de Hong Kong, un contexto cultural muy diferente al occiden- 
tal. Algunos autores han reportado que el interés generativo se encuentra muy influido por factores de tipo cultural (Hofer, Busch, Chasiotis, Kärtner, y Campos, 2007), lo que puede explicar las diferencias encontradas.

Así, los resultados sugieren que los mayores participantes en nuestro estudio, o al menos muchos de ellos, continúan teniendo un interés elevado por realizar contribuciones a los contextos sociales en los que participan, familiares o comunitarios y se implican en comportamientos que expresan ese interés. La expresión de este interés en forma de comportamientos como cuidar de otros, ofrecer experiencia y consejos, tratar de mejorar los entornos en los que viven o dejar un legado que les sobreviva es un aspecto frecuente en la vida de los mayores. De hecho, si se observan los comportamientos generativos incluidos en la escala ECG que presentan puntuaciones medias más elevadas, parece derivarse que en la vejez la generatividad que implica nutrir, beneficiar y cuidar de otras personas (lo que algunos autores denominan generatividad comunitaria, ver Kotre, 1984) es particularmente frecuente.

Los resultados también indican que en nuestra muestra de participantes mayores los comportamientos generativos (que no el interés generativo) tienden a ser menos frecuentes con la edad. Si esta reducción obedece a una decisión voluntaria para desvincularse del mundo que les rodea, o simplemente es producto de obstáculos (p.e. dificultades de salud, pérdida de relaciones, etc.) inherentes al proceso de envejecimiento es una cuestión que va más allá de nuestros objetivos y que podría dar pie a nuevas investigaciones. Los resultados obtenidos respecto a los dos indicadores de salud incluidos en nuestro estudio, sin embargo, no parecen indicar que este sea un factor que esté relacionado con los comportamientos generativos.

Estos intereses y comportamientos generativos en la vejez tienen, además, relación con el bienestar. En este sentido, los datos del estudio replican los obtenidos por McAdams et al. (1993) o Grossbaum y Bates (2002) utilizando muestras de mediana edad: mientras el interés generativo se relaciona con la satisfacción con la vida, las acciones generativas no. En este sentido, hay que tener en cuenta que la concreción de la generatividad en comportamientos efectivos se ve influida, además de por un interés previo, por diferentes circunstancias y obstáculos que en ocasiones pueden impedir que ese interés se transforme en comportamiento efectivo.

Considerar otras dimensiones del bienestar también podría ayudar a entender porqué, aparentemente, los mayores que más contribuyen de manera efectiva (mediante comportamientos generativos) no se benefician de mayores niveles de bienestar. La relación existen entre comportamientos generativo y orientación al futuro sugiere que sí obtienen esos beneficios, pero no en términos de bienestar hedónico (evaluado a partir de la satisfacción con la vida), sino en términos de bienestar eudaimónico (evaluado a partir de la orientación al futuro). De acuerdo con esta interpretación, los beneficios de comportarse generativamente no se recogen en forma de sensaciones placenteras inmediatas, sino más bien en la forma de tener la sensación de hacer algo con sentido y que nos permite sentirnos 'en desarrollo'. Sin embargo, esta relación entre orientación al futuro y comportamientos generativos quizá no es tan estrecha como el índice de correlación sugiere, dado que otros factores pueden estar detrás. De hecho, cuando tenemos en cuenta el peso predictivo los comportamientos generativos, en un análisis multivariable, el comportamiento generativo no sólo parece aportar poco a las puntuaciones de satisfacción con la vida, sino que tampoco aporta apenas nada a las de orientación al futuro. Sin embargo, el interés generativo sí confirma alto su poder predictivo respecto a la satisfacción con la vida. En cuando a la orientación al futuro, sólo la edad (en coherencia con estudios anteriores, como los de Lang y Carstensen, 2002 o Ryff, 1995) y en menor medida la salud subjetiva aparecen como predictores significativos.

La interpretación de los resultados obtenidos se ve sujeta, además, a las limitaciones del estudio. La falta de representatividad de la muestra es sin duda una de ellas. El método de selección de la muestra, no aleatorio, impide que los resultados puedan considerarse representativos de la población mayor. Por otra parte, al tratarse de un estudio correlacional, la dirección de las relaciones establecidas no puede asegurarse con certeza. Quizá son las personas que ya tienen mayor bienestar las que más interés generativo tienen y las que a su vez se implican más frecuentemente en comportamientos generativos. El uso de estudios de tipo experimental o cuasi-experimental (p.e., con medidas pre y post respecto a la implicación en ciertas tareas generativas) podría aportar datos relevantes respecto a esta cuestión. Por último, también cabe reseñar que la escala 'Orientación al futuro' quizá sólo abarca parte del significado atribuido al bienestar eudaimónico. El uso de otras medidas, o de múltiples medidas, ofrecería un panorama más preciso respecto a la relación entre la generatividad y este tipo de bienestar.

Pese a las limitaciones, el estudio aporta unas escalas psicométricamente adecuadas para evaluar interés y acción generativa en muestras de habla hispana y confirma la importancia que puede tener la generatividad en la vejez y sus complejas relaciones con el bienestar. Profundizar en estas relaciones o explorar el desarrollo de las diferentes facetas de la generatividad a lo largo de la vejez, y particularmente en la denominada 'cuarta edad' (Baltes y Smith, 2003), cuando la pérdida se hace mucho más presente, nos parecen vías de investigación futura particularmente prometedoras. En conjunto, este tipo de estudios contribuirán a consolidar una imagen de la vejez más positiva y alejada de estereotipos de pasividad y aislamiento.

Nota.- Este estudio ha contado con la financiación del Ministerio de Ciencia e Innovación, proyecto PSI2009-10966. 


\section{Referencias}

Baltes, P. B. y Baltes, M. M. (1990). Psychological perspectives on successful aging: The model of selective optimization with compensation. En P. B. Baltes y M. M. Baltes (Eds.), Successful aging. Perspectives from the behavioral sciences (pp. 1-34). Cambridge: Cambridge University Press.

Baltes, P. B. y Smith, J. (2003). New frontiers in the future of aging: From successful aging of the young old to the dilemmas of the fourth age. Gerontology, 49, 123-135.

Caro, F. G. y Sánchez, M. (2005). Envejecimiento productivo. Concepto y factores explicativos. En S. Pinazo Hernandis, y M. Sánchez, (Eds.), Gerontología. Actualización, innovación y propuestas (pp. 457-487). Madrid: Pearson Prentice Hall.

Cheng, S-T. (2009). Generativity in later life: Perceived respect from younger generations as a determinant of goal disengagement and psychological well-being. Journal of Gerontology: Psychological Sciences, 64B, 45-54.

Cumming, M. E. y Henry, W. E. (1961). Growing old: The process of disengagement. Nueva York: Basic Books.

Deci, E. L. y Ryan, R. M. (2008). Hedonia, eudaimonia, and well-being: an introduction. Journal of Happiness Studies, 9(1), 1-11.

Diener, E., Emmons, R. A., Larsen, R. J. y Griffin, S. (1985). The Satisfaction with Life Scale. Journal of Personality Assessment, 49, 71-75.

Erikson, E. H. (1950). Childhood and society. Nueva York: Norton.

Erikson, E. H., Erikson, J. M. y Kivnick, H. Q. (1986). Vital involvement in old age. Nueva York: Norton.

Fernández-Ballesteros, R. (2009). Envejecimiento activo. Contribuciones de la Psicología. Madrid: Pirámide.

Fisher, B. J. (1995). Successful aging, life satisfaction, and generativity in later life. International Journal of Aging \& Human Development, 41, 239-50.

Grossbaum, M. F. y Bates, G. W. (2002). Correlates of psychological wellbeing at midlife: The role of generativity, agency and communion, and narrative themes. International Journal of Behavioral Development, 26, 120127.

Hofer, J., Busch, H., Chasiotis, A., Kärtner, J. y Campos, D. (2007). Concern for generativity and its relation to implicit pro-social power motivation, generative goals and satisfaction with life: A cross-cultural investigation. Journal of Personality, 76, 1-30.

Kleiber, D. y Nimrod, G. (2008). Expressions of generativity and civic engagement in a 'learning in retirement' group. Journal of Adult Development, $15,76-86$.

Kotre, J. (1984). Outliving the self: Generativity and the interpretation of lives. Baltimore: Johns Hopkins University Press.

Lang, F. R. y Carstensen, L. L. (2002). Time counts: Future time perspective, goals, and social relationships. Psychology and Aging, 17, 125-139.

McAdams, D. y de St. Aubin, E. (1992). A theory of generativity and its assessment through self-report, behavioral acts, and narrative themes in autobiography. Journal of Personality and Social Psychology, 62, 1003-1015.

McAdams, D. P. y Logan, R. L. (2004). What is generativity? En E. de St. Aubin, D. P. McAdams y T. C. Kim (Eds.), The generative society (pp. 1531). Washington: American Psychological Association Press.

McAdams, D. P., de St. Aubin, E. y Logan, R. L. (1993). Generativity among young, midlife, and older adults. Pyschology and Aging, 8, 221-230.

McAdams, D. P., Hart, H. M. y Maruna, S. (1998). The anatomy of generativity. En D. P. McAdams y E. de St. Aubin (Eds.), Generativity and adult development (pp. 7-43). Washington, DC: American Psychological Association.

Miner-Rubino, K., Winter, D. G. y Stewart, A. J. (2004). Gender, social class, and the subjective experience of aging: self-perceived personality change from early adulthood to late midlife. Personality and Social Psychology Bulletin, 30, 1599-1610.
Moviñas, A. (1998). Representaciones de la vejez (modelos de disminución y de crecimiento). Anales de Psicología, 14, 13-25.

Naciones Unidas (2002). Madrid International Plan of Action on Aging 2002. Nueva York: Naciones Unidas. Recuperado de http://www.unngls.org/orf/pdf/MIPAA.pdf

Neugarten, B. L. (1977). Personality and aging. En Birren, J. E. y Schaie, K. W. (Eds.), Handbook of the Psychology of Aging (pp. 626-649). Nueva York: Van Nostrand Reinhold.

Organización Mundial de la Salud (2002). Active ageing: A policy framework. Ginebra: Organización Mundial de la Salud. Recuperado de http://whqlibdoc.who.int/hq/2002/WHO_NMH_NPH_02.8.pdf

Pons, D., Atienza, F. L., Balaguer, I. y García-Merita, M. (2000). Satisfaction With Life Scale: Analysis of factorial invariance for adolescents and elderly persons. Perceptual and Motor Skills, 91, 62-68.

Ranzijn, R. (2002). The potential of older adults to enhance community quality of life: Links between positive psychology and productive aging. Ageing International, 27, 30-55.

Rowe, J. W. y Kahn, R. L. (1997). Successful aging. The Gerontologist, 37, 433440.

Ryan, R. M. y Deci, E. L. (2001). On happiness and human potentials: A review of research on hedonic and eudaimonic well-being. Annual Review of Psychology, 52, 141-166.

Ryff, C. D. (1989). Happiness is everything, or is it? Explorations on the meaning of psychological well-being. Journal of Personality and Social Psychology, 57(6), 1069-1081.

Ryff, C. D. (1995). Psychological well-being in adult life. Current Directions in Psychological Science, 4, 99-104.

Sheldon, K. M. y Kasser, T. (2001). Getting older, getting better? Personal strivings and psychological maturity across the life span. Developmental Psychology, 37, 491-501.

Thiele, D. M. y Whelan, T. A. (2008). The relationship between grandparent satisfaction, meaning, and generativity. International Journal of Aging and Human Development, 66, 21-48.

Thornton, J. E. (2002). Myths of ageing and ageist stereotypes. Educational Gerontology, 28, 301-312.

Tornstam, L. (1996). Gerotranscendence: The contemplative dimension of ageing. Journal of Ageing Studies, 11, 143-154.

Villar, F. (2012). Successful ageing and development. The contribution of generativity in older age. Ageing \& Society, 32, 1087-1105.

Villar, F., Triadó, C., Celdrán, M. y Solé, C. (2010). Measuring well-being among Spanish older adults: Development of simplified version of Ryff's Scale of Psychological Well-being. Psychological Reports, 107(1), 265-280.

Warburton, J. y Gooch, M. (2007). Stewardship volunteering by older Australians: The generative response. Local Environment, 12, 43-55.

Warburton, J., McLaughlin, D. y Pinsker, D. (2006). Generative acts: Family and community involvement of older Australians. International Journal of Aging and Human Development, 63, 115-137.

Waterman, A. S. (1993). Two conceptions of happiness: Contrasts of personal expressiveness (eudaimonia) and hedonic enjoyment. Journal of Personality and Social Psychology, 64, 678-691.

Zucker, A. N., Ostrove, J. M. y Stewart, A. J. (2002). College-educated women's personality development in adulthood: Perceptions and age differences. Psychology and Aging, 17, 236-244.

(Articulo recibido: 24-1-2011; revisado: 26-9-2012; aceptado: 26-9-2012) 


\section{Anexo 1.}

Ítems de la Escala de Generatividad Loyola (EGL). Los ítems con puntuación invertida se marcan con asterisco.

1. Intento transmitir a otros el conocimiento que he ido adquiriendo a través de mis experiencias

2. Siento que nadie me necesita*

3. Creo que me gustaría hacer de maestro

4. Siento que importo a muchas personas

5. Evito ofrecerme como voluntario para colaborar en organizaciones sociales*

6. He hecho cosas que han influido bastante en otras personas

7. Intento ser creativo en la mayor parte de las cosas que hago

8. Pienso que, después de morir, seré recordado durante bastante tiempo

9. Creo que la sociedad no puede ser la responsable de proporcionar comida y vivienda a gente sin hogar*

10. Otros dirían de mí que he realizado importantes contribuciones a la sociedad

11. Si no fuera capaz de tener hijos propios, los habría adoptado

12. Tengo habilidades y conocimientos valiosos que intento enseñar a otros

13. Siento que nada de lo que he hecho sobrevivirá después de que muera*

14. En general, lo que hago no tiene un efecto positivo sobre los demás*

15. Me siento como si no hubiese hecho nada valioso que pudiese servir a otras personas *

16. A lo largo de mi vida me he comprometido con diferentes tipos de personas, grupos y actividades.

17. Las personas que me conocen dirían de mí que soy una persona muy productiva

18. La mejora de mi barrio es también responsabilidad mía

19. La gente viene a mí a pedirme consejo

20. Creo que mis contribuciones se mantendrán después de que yo muera 


\section{Anexo 2.}

Ítems de la Escala de Comportamientos generativos (ECG)

En los últimos dos meses...

1. He visitado a una persona en el hospital o en una residencia

2. He realizado alguna actividad manual o artística (como alfarería, bordado, pintura, etc.)

3. He transmitido valores a personas más jóvenes

4. He donado dinero a una causa benéfica

5. He tenido a mis nietos a mi cargo

6. He dirigido a un grupo de personas para conseguir un bien común

7. He continuado desempeñando mi profesión

8. He contado a alguien experiencias personales que considero valiosas

9. He atendido las necesidades de personas que me rodean

10. Me he comprometido en asuntos políticos, sociales o religiosos

11. He dado dinero a personas de mi familia, o a amigos

12. He ayudado a personas allegadas con problemas

13. He arreglado cosas de la casa (una máquina, un mueble, un instrumento...)

14. He enseñado una habilidad o conocimiento a alguien

15. He echado una mano a otras personas en su trabajo

16. He cuidado de plantas o animales domésticos

17. He organizado un viaje, una fiesta o un evento social

18. He participado en reuniones de la comunidad o del barrio

19. He aconsejado a otras personas sobre decisiones que tenían que tomar

20. He cuidado del bienestar de mi familia

21. He regalado a un familiar o amigo algo que necesitaba

22. He dado limosna a personas que lo necesitan

23. He escrito relatos, poesías o memorias

24. He ayudado a mis hijos en tareas cotidianas

25. He actuado como mediador en conflictos de personas de mi entorno

26. He aprendido alguna habilidad o conocimiento útil para mi vida cotidiana

27. He realizado acciones que ayudan a mejorar el medio ambiente (reciclar, plantar un árbol, recoger basura de la calle, etc.)

28. He participado en una asociación u organización como voluntario

29. He cuidado a personas dependientes 